مجلة العلوم التربوية والإنسانية

Journal of Educational and Human Sciences

www.jeahs.com

Volume (5) April 2021

العدد (5) أبريل 2021

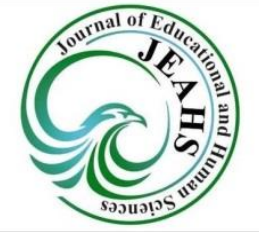

\title{
The Authority between The State of Law and The Chaos of The Conflict (A comparative study)
}

\section{Le pouvoir entre l'état de droit et le chaos de conflit (Etude comparative)}

\author{
Dr. Hussein Kiliban Ali Al Bareh \\ College of Imam Al-Aazam University - Iraq \\ Email: h.k.a.19621962@gmail.com
}

\begin{abstract}
In this comparative study, warning signals to the Arab Muslim community on the chaos of the political approach pursued and directed by a global hegemonic political force, the peoples have stood before it, losing power and will ... and in it an explanation of how the Western political approach developed two centuries of time after chaos prevailed between the interests of the authority and the goals of the church ... and democratically the concepts of power crystallized in this current profession, which is in line with the Western peoples .... On the other hand, how did the style of power among Arab Muslims descend to below over the past hundred years of political chaos we do not lose sight of that global hegemony that has been the reason for its manufacture and formulation over the years and with a long, discreet and thoughtful spirit through leaders who create chaos and reach the desired goal .
\end{abstract}

Keywords: State of Law, Chaos of The Conflict. 


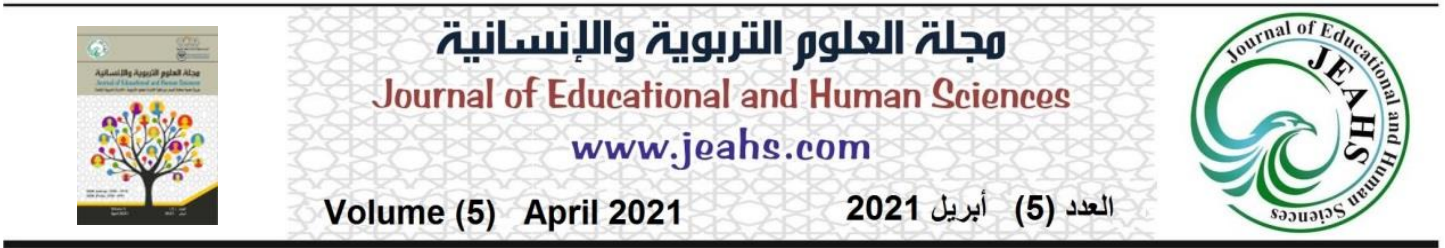

\section{L'introduction}

La raison de la transformation de la société occidentale vers un état de loi et d'institutions et la transformation de la société arabe À un état de conflits et de lute est est une raison pour laquelle "l'État" en Europe est passé d'un État du (roi / empereur) à un État moderne (état de modernité politique), État de droit et des institutions, un État qui tire sa légitimité de sa capacité à représenter la volonté du peuple exprimée par des élections libres, et qui œuvre au service de l'intérêt public. Au cours du Moyen Âge, pourquoi l'État n'a-t-il pas évolué pour devenir un État moderne de ce type, puis les tentatives des élites modernes ont ensuite échoué du pays de l'Islam pour transférer ces (modernités politiques) occidentales dans leurs pays...

\section{La problématique de la recherche et ses objectifs:}

Lorsque nous posons la question (pourquoi) sur ce cas politique, nous ne voulons pas appliquer le modèle européen, mais nous voulons révéler la spécificité de ce modèle en le comparant avec le modèle connu dans les pays de l'Islam, et en comparant les deux modèles en partant du principe que l'un est pareil avec l'autre, et non en partant du principe que l'un est l'origine et l'autre est la branche, et pour connaitre bien le modèle européen à partir de la lecture du modèle islamique, la particularité de ce dernier est clairement mise en evidence, et en appliquant cette approche, le sujet révèle clairement que l'Etat européen modern n'est que l'état des institutions et l'intérêt suprême (par l'opposition à l'État tyran d'une part et aux intérêts du clan ou de la secte d'autre part)...

\section{Le prince et l'église, un conflit politique:}

Il est apparu en Europe à la suite de processus historiques qui ont abouti à l'émergence d'une nouvelle sphère de la vie sociale: la sphère politique, à savoir la pratique politique que (le Prince) rivalise avec (l'Église) et se sont présentés comme une alternative à la vie politique...

Cette sphère est née à cause du conflit entre le prince et l'église au Moyen Âge, ce conflit qui a abouti à l'émergence de la théorie du contrat (ou le contrat social - avant Rousseau), qui dit: que l'autorité de l'Église est d'Allah, et donc supérieure, tandis que l'autorité du prince est du peuple et par conséquent elle est le plus bas.

Cette répartition dans la source du pouvoir aboutit à dire que le Prince peut être isolé s'il ne travaille pas au profit de l'intérêt public, est donc apparu comme un élément nouveau, mais comme une tierce partie dans l'arène du conflit qui est (la population), (l'intérêt public) et (la constitution de la légitimité du pouvoir à contracter), qui est devenue une nouvelle sphère (politique)...

C'est ce qui s'est passé en Europe, mais les pays islamiques ne connaissaient pas un tel processus historique et ne connaissaient donc pas de rupture similaire entre le 


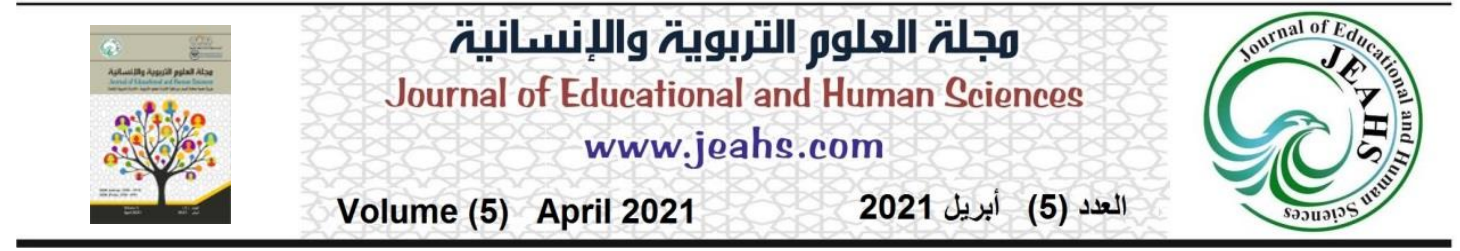

(Prince) et ce qu'on appele dans ces pays l'(État), mais toujours reste le (Prince) comme on dit l'(État) et l'État reste toujours le Prince, mais pourquoi qu'il n'ya pas d'église dans l'islam, c'est pourquoi l'histoire des pays arabo-musulmans est dépourvue du conflit que l'Europe a connu entre l'église et le prince, et cela était dans le passé, mais quant au présent quand les élites modernes dans les pays araboislamiques ont échoué (d'importer) la modèrnité politique occidentale et de l'enraciner dans leurs territoires, revient en raison du facteur majeur qui est la sphère politique dans ces pays comme celle du Moyen Âge, il s'agissait d'une sphère occupée uniquement par le (prince) et gardée bien par la religion, ce qui explique, à notre avis, que les mouvements interdictionnels observés dans le monde arabe et islamique sont dirigés à la fois contre le Prince et contre les élites modernes, leurs gouvernements et leurs parlements; Parce que c'est l'échec de toutes les idéologies transférées et importees de la modernité politique européenne, le public n'a plus que l'Islam et les prédicateurs n'ont autre moyen de mobiliser les masses que l'islam, d'où vient le (réveil islamique)...

Donc le conflit en Europe était entre l'église et le prince et l'absence de ce conflit dans les pays musulmans, ce type de mesure n'est pas fiable, et cela ne peut pas être prouvé, et cela nous rappelle la théorie de Max Weber sur l'émergence du capitalisme en Europe, il croit que l'éthique protestante qui s'est répandue avec la réforme religieuse par (Martin Luther King) au XVIe siècle, qui prônait à l'action, au gain, et à l' économie, même l' économie dans la dépense c'est elle qui était derrière la propagation de ce qu'il a appelé «l'esprit capitaliste» fondé sur la recherche du profit, sur la planification et le comportement rationnels, c'est que le capitalisme n'apparaissait pas dans le monde arabo-islamique; car aucune réforme de la religion du genre d'Europe n' y était connue ...

On peut expliquer l'émergence de la modernité politique en Europe et le développement de conditions économiques dues à la cause de forces sociales internes, sans aucune ingérence ni influence d'aucune force externe susceptible d'entraver le processus de développement...En ce qui concerne le monde arabe et islamique, le facteur externe a été l'une des principales raisons de la détérioration de la situation dans ce pays: ce facteur externe est représenté par l'invasion directe et destructrice sur la région arabe, ainsi que par le concept du champ politique pour souligner tout d'abord sa signification procédurale et qu'il n'ya aucune voie unique pour émerger la sphère politique dans les societies, ce n'est pas seulement un conflit entre l'église et le prince, mais aussi la source était du peuple (le contrat social, l'intérêt public), c'est un des plusieurs cas, un état qui a été développé par un certain nombre de facteurs en un état d'institutions contemporaines que l'histoire n'a jamais vu auparavant, mais la sphère politique comme un domaine dans lequel la politique pratique son rôle sans l'église, un phénomène connu par plusieurs expériences historiques, y compris l'expérience de la démocratie grecque d'une part et l'expérience de (Mouawia) dans la civilisation arabo-islamique d'autre part... 


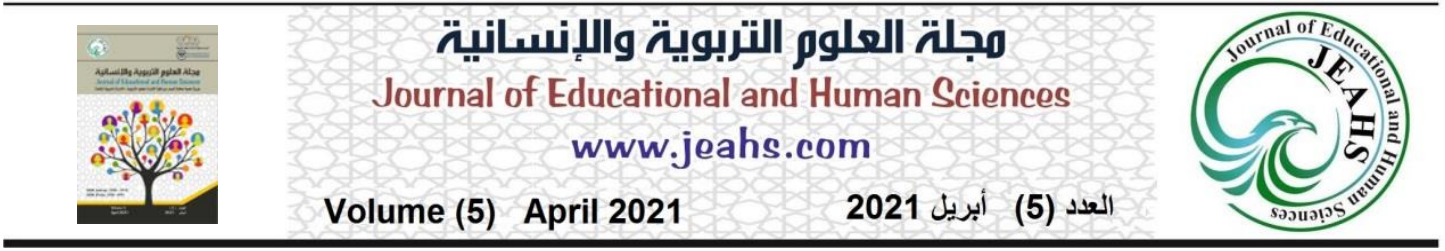

Ce qui nous importe ici, c'est de noter que ce qui distingue le champ politique qui a émergé en Europe avec le système capitaliste dans lequel il prévaut et qui est clairement divisé en deux structures: l'infrastructure ou la base économique, que l'industrie forme son ossature, et une superstructure que ses organs sont des institutions de l'État, l'idéologie qui y est associée, mais la société arabe ne connaissait pas cette distinction claire entre les deux structures, et ce qui apparu principalement est le chevauchement de leurs éléments, ce qui fait la société tout entière est comme une structure unique.

La force motrice de ces sociétés réside dans leur intérêt pour le domaine économique: elles se distinguent les unes des autres par leur honneur, leur prestige, leur argent ou leur artisanat, et que ces sociétés sont orientées invisiblement par leurs intérêts économiques, mais au niveau des pays arabes et islamiques, le mouvement s'oriente vers: le tribalisme, le sectarisme et la solidarité familiale, les gens ici sont soulignés par leurs intérêts économiques, mais restent influencés par les idées de la tribu ou de la secte, ainsi que par le chevauchement de données qu'il est difficile de distinguer entre ce qui est une règle et ce qui est un reflet. En d'autres termes, le conflit social dans les sociétés anciennes capitalistes ne prend pas la forme d'une réflexion sur la réalité économique des groupes en conflit, mais plutôt la continuation de conflits sur lesquels nous avons une base économique et sociale passée et non présente.

Après ces observations méthodologiques, Bano souligne l'unité de l'infrastructure et de la superstructure dans les sociétés extérieures, affirmant que (la vie privée de la société, qui est de type externe, est la relation dialectique qui existe entre l'unité de l'État et la société, que cette unité est fondée par les tâches économiques de l'État, et le conflit social qui existe entre l'aristocratie et les paysans et les deux parties de cet argument (l'unité de l'État /la société d'un côté, et le conflit entre l'aristocratie et les paysans de l'autre côté, se reflète ensemble, dans l'idéologie de cette société, formant un ensemble dense de développements et de concepts contradictoires).

En d'autres termes, le conflit idéologique dans la société d'esclavages (Athènes / Rome) ne reflète pas la lutte de classe entre les citoyens et les esclaves, mais reflète les rivalités et les différences d'intérêts au sein de la classe des citoyens eux-mêmes. Il existe donc un décalage entre les deux relations contradictoires, un conflit social entre les esclaves et les citoyens et le conflit intellectuel au sein de la classe des citoyens eux-mêmes, et selon Bano les rivalités et les différences philosophiques n'aient atteint, à Athènes ou à Rome, jusqu'au plus profond de la contradiction sociale, ne nous posant aucun problème réel d'exclusion de l'homme à son prochain.

Telle est la situation dans le système esclavagiste, mais dans les sociétés fonctionnelles, la situation est différente, car de nombreux paysans constituaient le centre d'inspiration de certains courants philosophiques et ne constituaient pas des postes occupés au sein de la même aristocratie qu'en Grèce...La critique dans cette 


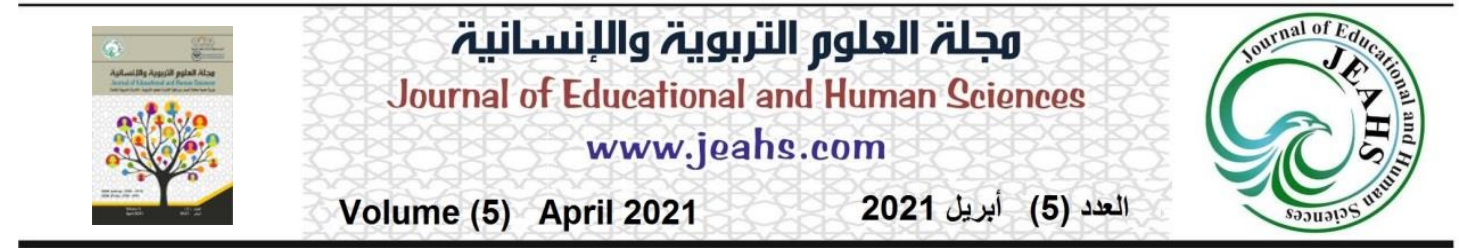

classe est une critique de la façon dont ceux qui sont au sommet de la société et du pouvoir d'accomplir leurs tâches.

Nous trouvons ici la différence entre les manifestations d'esclaves dans le système esclavagiste et les manifestations de paysans dans le régime fonctionnelles: dans l'esclavage où l'esclave n'a aucun droit légal chez son maitre, quand ils se révoltent, c'est pour abolir leur statut d'esclaves, c'est-à-dire comme personnes privées du droit, mais ils rêvent souvent de devenir maîtres et ont des esclaves... Selon Bano, dans les anciens textes orientaux:nous trouvons le conférencier est un homme qui a des droits, y compris des litiges, il se plaint que ses droits ont été violés, bien qu'il ait le droit de porter l'affaire devant le pouvoir judiciaire, mais il est en vain d' exercer ce pouvoir, le juge prononce toujours un jugement injuste, ainsi qu'il aspire donc à plus que "la justice", ce qui signifie d'améliorer sa situation tout en préservant le statut de la collectivité à laquelle il appartient et les devoirs de l'État envers "l'importance de l'exigence de justice."

Nous trouvons ici des observations tirées de l'étude des textes orientaux anciens, babyloniens et égyptiens pharaoniques, qui poussent la pensée et la vision de la libération du dogmatisme du matérialisme historique, (officiel), qui ne fait des idéologies que des reflets ou expressions de la situation de classe.

La relation entre la pensée et la réalité se diffère de ce que nous avons vu d'une société à l'autre: il est certain que le lecteur familier avec la littérature politique royale de l'héritage arabe trouve ce type d'expression plus crédible que celui utilisé par certains marxistes arabes qui interprètent tous les types de relations entre la pensée et la réalité à travers le matérialisme historique, ils prennent de ce dualisme matérialiste / idéaliste comme la clé du problème du rapport entre la pensée et la réalité à toutes les époques, ils font de tout ce qui est matériel en termes d'idéologie une expression des classes opprimées et de tout ce qui est idéal en elle comme expression du pouvoir des classes qui pratiquent la persécution. C'est une fausse clé celui qui ouvre les portes, alors comment faire ce type de clé si elle les ouvre tout le temps?

\section{La comparaison de Michel Foucault entre l'ancienne pensée orientale et la pensée grecque:}

Dans ce contexte, nous présentons une comparaison présentée par Michel Foucault entre le contenu de l'expression "pasteur et paroisse" dans l'ancienne pensée orientale et le contenu qui était dans la pensée grecque, et cela dans le cadre de l'appel à une critique radicale de l'autorité de l'État et de sa logique dans l'europe moderne, nous nous intéressons à cet aspect, qui concerne le vieil Orient car il complète l'image que nous avions connue auparavant. Foucault note que la perception qui fait du roi ou du président comme un (berger) parraine les (moutons) (les termes utilisés dans le texte français sont: moutons, bétails, troupeaux).Mais nous 


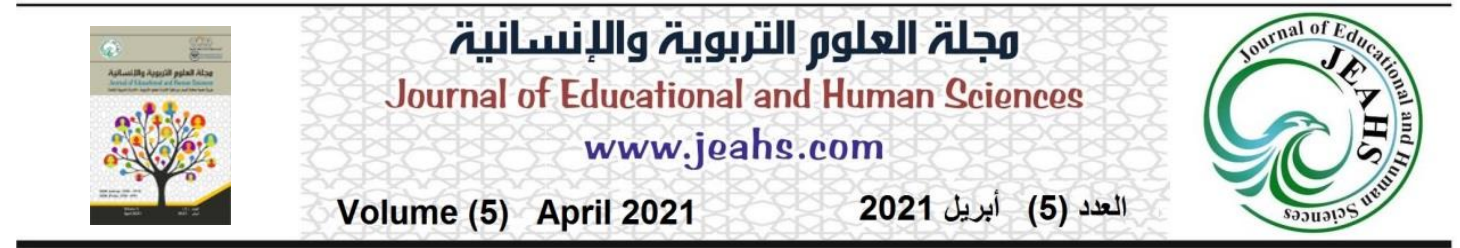

utilisons le mot arabe "paroisse", qui est déjà utilisé en: bétails, pastoral ou le berger (voir la langue des Arabes "Lissan Al arab"). Ce n'est pas une perception commune dans la pensée grecque ou romaine, bien que certains textes soient attribués à Homère, mais qu'ils soient totalement absents des textes politiques de base de Rome et de Grec. Alors que nous trouvons ce sentiment très commun dans les civilisations de l'ancien Orient et en Égypte, en Assyrie et en Palestine, Pharaon, par exemple, était considéré comme un berger et prenait le bâton du berger dans le cadre de rituels spéciaux pour son couronnement, tandis que le roi de Babylone devait être appelé (berger du peuple) parmi d'autres titres.

Le titre de berger n'était pas propre aux rois, mais ainsi pour Dieu: les Babyloniens considéraient leur Dieu comme un berger, conduisant les gens au pâturage et les nourrissant. Foucault ajoute que la combinaison du roi et du dieu ici en appelant le berger c'est quelque chose imposée pour ces deux ensembles, car ils jouent tous les deux le même rôle...Des Babyloniens et des Égyptiens anciens, Foucault se refère aux Hébreux pour constater qu'ils ont étendu l'usage du concept de berger et de paroisse, ajoutant à son usage une particularité distinguée: celui qui Le guide - pour eux c'est Allah - Il est Le Seul Berger de son peuple, lorsque Allah a ordonné David de recueillir le troupeau (les enfants d'Isrel), Il a établi un pouvoir et est devenu rénommé un berger... Sur la base de ces observations, Foucault souligne la différence entre le "pouvoir pastoral" dans l'Orient ancien et la pensée politique en Grèce, à travers les comparaisons suivantes :

A-On remarque tout d'abord que le berger de la pensée orientale ancienne exerce son autorité sur la paroisse (troupeau) et non sur le terrain comme dans la pensée grecque. Ainsi, la relation entre les dieux et les humains est différente ici et là: les dieux dans les Grecs sont propriétaires du pays, c'est-à-dire que la relation des dieux avec les hommes passe par la terre...Mais dans la pensée orientale antique, il n'ya pas d'(intermédiaire) dans la relation entre le dieu berger et son troupeau, cette relation directe liée au dieu berger qui donnait la terre à son peuple (la terre promise des enfants d'Israël), et dans un autre sens: les dieux de la pensée grecque possèdent la terre et se comportent par eux dans le destin du peuple, mais dans l'ancienne pensée orientale, le dieu a des esclaves et peut leur donner la terre et leur la promettre.

B-La mission du berger est de réunir et de diriger la paroisse.Il ne fait aucun doute que la mission politique du président grec est d'assurer le calme et la sécurité dans la ville et de répandre l'unité et la coalition, mais la différence est Claire dans cette question entre la pensée orientale antique et la pensée grecque, ce que le berger oriental recueille, ce sont les individus dispersés qui répondent à son appel s'il les invite à rencontrer par son cri ou son sifflet, mais une fois que le berger a disparu, le troupeau / la paroisse se disperse, car la rencontre dépend du berger et cette situation est différente pour la Grèce, alors le sage Solon le vertueux, qui a séparé les conflits qui existaient entre les habitants d'Athènes sont allés et ont laissé (une ville) un État 


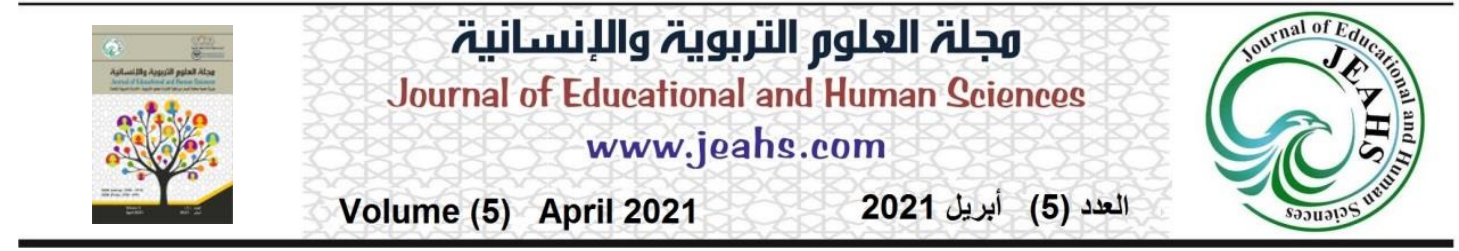

fort doté de lois lui permettant de rester après lui.

C-Le berger dans la pensée orientale garantit le salut pour la paroisse, et les Grecs disent également que les dieux sauvent la ville, et comparent souvent le président vertueux au capitaine de son bateau qui s'éloigne des rochers pour éviter de les toucher, mais le moyen de berger pour sauver sa paroiss-à la pensée oriontale- était différente de la façon dont le maître sauvait son navire: alors le «soins» ne signifie pas ici la direction du navire et d' éviter les dangers en tant que navire, et uniquement contre les dangers, mais constitue un soin permanent du berger pour chaque individu de sa paroisse.

D-Foucault met en évidence une autre différence cette fois concernant l'exercice du pouvoir et ses motivations: le président, c'est le président de la ville aux Grecs, ses décisions doivent être prises au profit de tous, où il s'acquitte de son devoir, son devoir de président, qui l'impose, mais s'il s'éloigne de cela ou s'il privilégie ses intérêts personnels, il est un mauvais president. Alors ceux qui sacrifient leurs vies pendant la guerre, pour la ville, sont des héros vraiment immortels, mais le berger est l'issu de la sincérité dans ses soins envers sa paroisse, ce n'est pas pour l'idée du devoir...Foucault, dans ses remarques précédentes, a déclaré: Je ne dis pas que le pouvoir politique était effectivement exercé de cette manière dans la société juive avant la chute de Jérusalem (Aux mains des Romains), mais ce que je veux souligner, c'est que ce type de perception de relation entre le berger et la paroisse s'est largement répandu au Moyen Âge, et l'époque moderne et d'une grande importance, Foucault poursuit son analyse des types de perception qui prévalaient en Europe à propos du pouvoir pastoral et, à l'époque moderne, à propos du pouvoir basé sur l'idée de (logique étatique), il est noté que le concept formel de pasteur et de paroisse ayant transféré vers l'Europe par le christianisme, et non par le concept grec, et que l'autorité a donc fait dans la société européenne une technique étrange consistant à traiter la grande majorité de la population comme un troupeau dirigé par plusieurs bergers, ce qui constitue une rupture complète avec la (ville) grecque, de sorte que le caractère politique raisonnable est devenu complètement différent de ce qu'il était en Grèce, l'idée alors du pouvoir pastoral a enraciné au Moyen Âge, Vient ensuite l'idée de la logique de l'État (à l'ère moderne) et de l'idée et de la logique de l'État à ses résultats inévitables: la consolidation et l'assemblée: la consécration de l'individualisme et en même temps l'exercice de la domination universelle et totale...Foucault termine son discours en s'attaquant aux racines du raisonnable politique lui-même, en critiquant les fondements de la pratique politique de l'État, que l'esprit politique de chaque époque et de chaque civilisation cherche à justifier, à légitimer et à rationaliser, et si Foucault a terminé de cette équilibre dans le concept de berger et de paroisse à dire que les démocraties occidentales contemporaines cachent derrière leurs manifestations prestigieuses une(technologie occidentale) dans l'exercice du pouvoir composé d'une poignée de pasteurs dirigeant la grande majorité de la population et les traitent comme un troupeau. Nous ici, dans le monde arabe et les sociétés orientales n'ont pas besoin d'un telle équilibre pour montrer comment la 


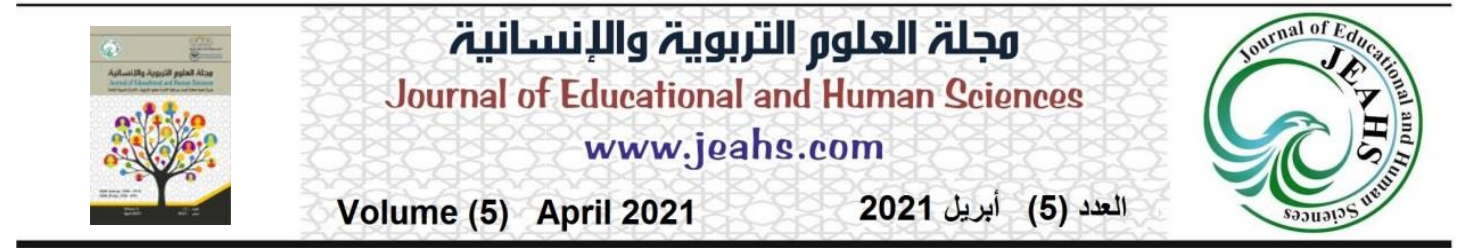

réalité de notre puissance, en apparence et en essence reposant sur une technique qui nous est familière, technique d'un seul berger menant toute la paroisse, il suffit de noter en retour que nous oublions à cause de la pensée d'intrus que (paroisse) signifie en arabe les troupeaux, et un peu ce que nous alertons que le berger chez nous est un, ainsi que le souverain voit pour participer au gouvernement: il est toujours considéré comme une piété et une association politique malgré le slogan du (pluralisme) brandi ça et là, que le pluralisme est sans valeur si ce n'est pas l'institution du partage, le partage du pouvoir et de sa circulation.

Ainsi, lorsque les résultats de la jurisprudence occidentale contemporaine, qui soulignent le phénomène social total dans les sociétés non capitalistes et l'infrastructure et la superstructure qui s'y trouvent, et l'importance du rôle de la religion et de la parenté, ainsi que du facteur économique, qui diffèrent par le degré de détermination et de résolution d'une société à une autre et d'une période historique. À un autre...Lorsque nous le faisons, nous ne choisissons pas ces assurances comme le pensent les proprietaries, et nous en tenons compte dans les données qu'ils en ont prises...Mais nous le prenons parce que cela nous rapproche de notre patrimoine, parce que cela nous ramène à Ibn Khaldoun, mais pas pour révéler l'importance de ses points de vue et pour peser l'équilibre des théories occidentales modernes, comme beaucoup d'entre nous, mais pour révéler cette fois-ci notre réalité socio-politique contemporaine, la présence des mêmes déterminants analysés par Ibn Khaldoun et en a fait le sujet d'une nouvelle science qu'il a appelée (architecture humaine), une présence non sous la même forme dans la même image, mais en présence d'un actif en tout cas....Le retour à Ibn Khaldoun est justifié comme suit: ${ }^{(1)}$

Premièrement: La réalité sociale et politique actuelle, qui existe dans le monde arabe et dans d'autres pays, fait que parler du clan, du sectarisme et du fondamentalisme religieux a récemment été autorisé ou même requis, une parole non (rétroactif) ou déprécié, comme c'était le cas il ya deux décennies...S'il est vrai que (l'analyse du passé nous fournit les clés du présent), comme le dit Marx, notre présent arabe, qui est connu sous le nom de "retour refoulé" clanique, sectaire et de l'externalité religieux, nous présente certaines des clés du présent, sinon ses clés, qui nous dirigent vers la pensée d'Ibn Khaldoun. Parce que c'est la même chose qu'il avait déjà apprise de son présent, de sa riche expérience politique dans laquelle il a également découvert (les clés du présent) repentant en écho, (le présent est comme l'avenir de l'eau à l'eau).

Le tribalisme e et la prédication religieuse ne sont pas toutes les clés qu'Ibn Khaldoun nous offre à propos de l'histoire arabo-islamique, mais il y a une troisième clé qui était constamment présente dans la pensée de l'auteur de l'introduction, bien

( ${ }^{1}$ )Ibn Khaldoun, Abd al-Rahman ibn Muhammad ibn Khaldoun, Enquête sur Abdul Wahid Wafi, 4 e s. (Comité de la déclaration arabe du Caire, 1908), 1/364. avait sa proper justification... 


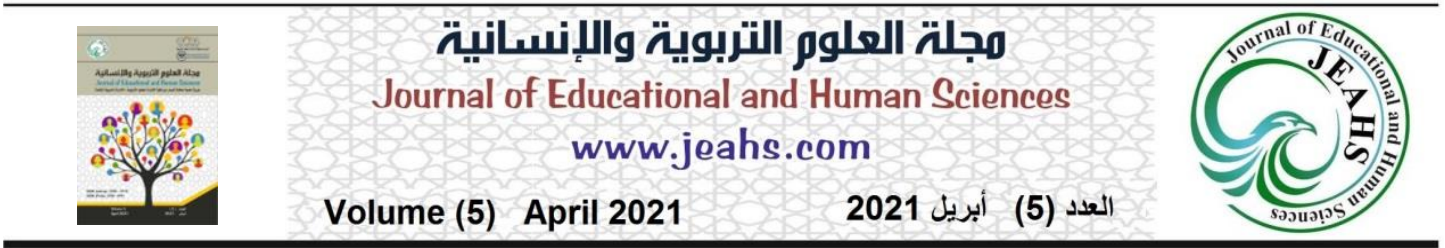

qu'il ne lui ait pas donné de nom spécial: c'est le facteur économique qui ne s'est pas présenté à l'époque d'Ibn Khaldoun, une époque précapitaliste, qui joua néanmoins un role, parfois et derrière un rideau (decisive), nous avons mis en évidence ce facteur et l'avons appelé (méthode de production de l'économie fondée sur l'invasion), c'est-àdire, d'extraire le surplus de la production par la force. Ibn Khaldoun l'a décrite comme une (doctrine de la vie anormale), car elle ne repose pas sur la production, mais dépend de l'invasion, à la fois à l'intérieur et à l'extérieur. En d'autres termes, (l'État recueille de l'argent de la paroisse et le dépense pour ses proches membres et ses hommes, c'est à dire, que (l'Etat a recueilli des fonds de la paroisse et sont allés aux hommes de l'état qui sont la majorité, leur richesse et les rendements de luxe donc se glorifieront et s'augmenteront de plus en plus $)^{(1)}$

Ce type d'économie s'appelle (économie rentière) ${ }^{(2)}$... Le comportement tribal, et l'extrémisme religieuse, trois (clés) ou déterminants qu'on ne peut pas résoudre les mystères actuels arabes sans eux, et ce sont les mêmes (clés) employés par Ibn Khaldoun dans son lecture de l'expérience de la civilisation arabo-islamique de son époque...

\section{La relation entre le réalisme d'Aristote et la pensée arabo-islamique:}

Le réalisme, qui est un terme philosophique arabe, et la pensée arabo-islamique (représentée par Muhammad Abdo) est un penseur islamique moderne...Le lien entre les deux hommes et les deux termes est une vue sur la question du point de vue du problème de (la réforme politique) comme un problème éternel opposant et opposant toute personne qui pense à la manière d'établir une sorte de relation fondée sur la justice et l'équité entre les citoyens en général et entre les gouvernants et les gouvernés en particulier, ce problème (Le problème politique) est en effet a apparu depuis que l'Etat est devenu comme un corp et une autorité suprême, représente-les et agisse en leur nom ou sans leur consentement, malgré cela on peut dire selon l'histoire de la pensée politique: que la première présentation théorique profonde des aspects de ce problème et de ses dimensions a été trouvée par les philosophes de la Grèce, en particulier Platon et Aristote, et ce qui nous préoccupe ici est que le cœur du problème était discuté par Platon et par son élève Aristote d'un coin différent, et que les deux points de vue opposés depuis long temps, nous les trouvons se battre sous la (robe de Mohammed Abdah) au début du XXe siècle et dans les cœurs et les esprits de nombreux hommes de ce XXIe siècle, c'est que Al Cheikh Mohammed Abdah a trouvé à la fin de son tour politique et de la réforme une resolution de cette problème dans ce terme qui rassemble deux sujets opposés, c'est le terme " le juste

( $\left.{ }^{1}\right)$ Jabri, la pensée d'Ibn Khaldoun, la désobéissance et l'État, les caractéristiques de la théorie de Khaldouni dans l'histoire islamique, p.872

$\left({ }^{2}\right)$ Sur l'économie rentière et l'état rentier, voir La nation, l'état et l'intégration dans le monde arabe, de Ghassan Salameh (et al.), C2 Beyrouth: Centre d'études sur l'unité arabe / 1989 


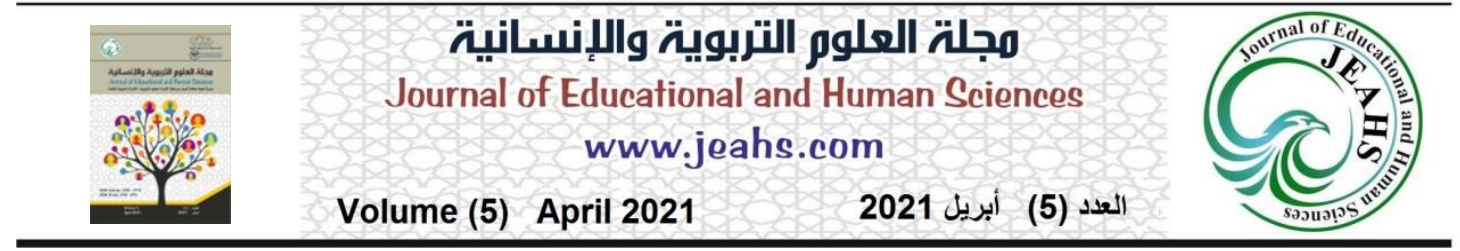

despote" qui fait référence à Platon, dont l'analyse et la discussion sur la question de la gouvernance équitable est terminée, pour dire: il ne convient pas à la société sauf si le chef de l'État est un sage philosophe, et c'est le même point de vue de Muhammad Abdah. Sans rien avoir à faire avec Platon, et il a rejeté le point de vue d'Aristote, qu'il a répondu à Platon, son professeur, en soulignant que la réforme ne repose pas sur une personne, aussi sage et juste qu'il ne garantisse pas que celui qui le suivra le sera. Aristote devant cela croyait que le plus sûr est la loi et non la personalité...

\section{Et les détails sont les suivants:}

Platon a fondé sa théorie politique sur la similitude entre les forces de la psyché humaine, qui en a trois: une force lubrique, le statut du grand public dans la société (leur vie est limitée pour satisfaire leurs besoins en nourriture et en boisson), une force nerveuse dont le statut est celui de soldats et de gardes (dont la tâche est de protéger et de défendre la société), ainsi qu'une force rationnelle est un statut d'hommes de pensée et de philosophie, qui utilisent le savoir, cherchent la vérité et mettent en valeur les meilleures valeurs et idéaux, puisqu'une personne n'est vertueuse que si sa force opprimante y brise sa force sexuelle, donc la cité vertueuse exige que le mot suprême soit pour la force rationnelle qui restreigne le pouvoir de colère et le met au travail pour réduire les convoitises de pouvoir lubrique, et établit l'équilibre entre eux, en d'autres termes la ville vertueuse ne peut être une utopie que si (l'esprit) assume la tâche de gouvernance et de gestion, et l'esprit à ce niveau sont sont des philosophes qui sont préparés pour acquérir les qualifications morales et intellectuelles qui leur permettent d'apprendre la philosophie et pour devenir des dirigeants philosophiques (vont avec ce que les gens cherchent aujourd'hui à honorer, et le considèrent comme trivial et inconvenient pour un homme libre. Ils ne tiennent qu'à l'accomplissement de leur devoir et à l'honneur qui en découle et à la justice qu'ils considèrent comme la chose la plus importante et la plus nécessaire à leur service, et ils évaluent souvent leur ardeur pour que leur (ville soit organisée).

Platon avait étudié les types de gouvernance jusqu'à son époque et les avait classés en trois catégories:

1-La gouvernance d'un individu: Peut-elle être vertueuse, c'est la gouvernance du philosophe, mais elle peut être déviée, puis deviendra tyrannique.

2-La gouvernance de la minorité: qui peut être valide, c'est la gouvernance de l'aristocratie ou le gouvernement des élites, et devant cela le gouvernement de la minorité invertueuse, la gouvernance oligarique, un gouvernement des riches.

3-La gouvernance de la majorité: peut être valide par sa constitution, et peut être invalide sans la loi, c'est la gouvernance de mob (Al gaouga).

Aristote a discuté ces trois catégories de gouvernance et préféré entre elles, en commençant par la rédaction d'un critère basé sur la preference, et la norme ici (la loi) 


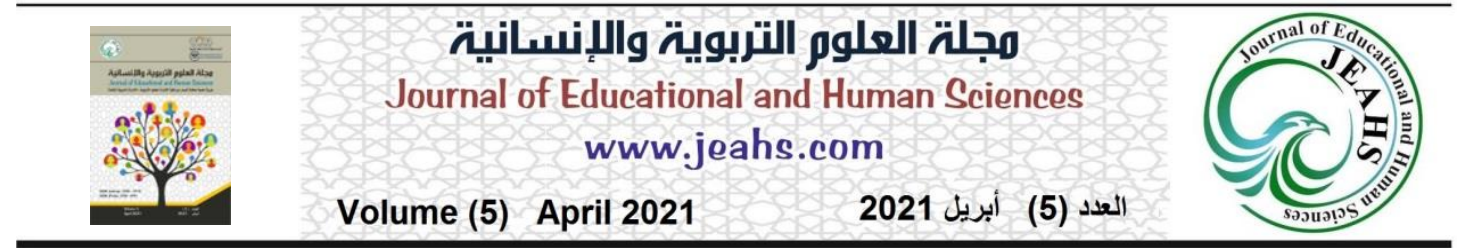

ou (l'honneur personnelle), et dans ce domaine, Aristote a décidé la nécessité de recourir à la loi, qui était supérieure à quiconque, qu'il soit philosophe ou non, et il justifie que la relation entre le souverain et le peuple est une relation de pouvoir entre une partie l'exerce et une autre partie à laquelle elle est soumise, cette relation diffère de celle d'une autre soumission telle que celle entre le père le chef de famille et les enfants, l'épouse et les domestiques, ou entre messieurs et esclaves, l'État donc selon Aristote, n'est pas une grande famille, mais un groupe de citoyens, et l'État ne fait pas partie de la relation entre les messieurs et les esclaves, mais la relation entre l'État et les citoyens est un partenariat, car l'État est (un corps social composé de membres égaux, ils partagent leur désir commun de mener une vie la plus en forme possible), Aristote a ajouté: "Les meilleures lois valent mieux que le meilleur des hommes" parce que les meilleurs hommes ne peuvent pas se passer de la loi, mais la préférence de Platon pour "les meilleurs hommes" se base sur l'analogie du président avec le médecin qui guérit les patients, c'est une vue implique une grosse erreur chez Aristote...

Alors que la relation entre le dirigeant et les citoyens est une relation politique et vise, comme nous l'avons dit, à réaliser l'égalité et la liberté, et cela ne peut être réalisée en abandonnant les responsabilités des citoyens comme le fait le patient.

L'opinion publique, qui est rationnelle, rarement commet des erreurs tandis que le plus sage des dirigeants voit souvent l'erreur. C'est donc vraiment une bonne gouvernance, dit Aristote, que le dirigeant base sur la loi.

Selon Aristote, le règne individuel du roi vertueux, comme l'a imaginé Platon, le philosophe, est sans aucun doute une règle vertueuse, comme dit Aristote, mais ce roi philosophe sera aussi la loi lorsqu'il prendra lui-même le pouvoir ou, dans ce cas, sera un dieu parmi les humains.

En ce qui concerne le problème politique qui nous préoccupe ici, les libéraux ont soulevé le slogan de la démocratie, alors que les réformateurs salafistes ont appelé à la shura, tandis que Cheikh Mohammed Abdah discute (le problème politique) se situe à mi-chemin entre Platon et Aristote, mais sans faire aucune pensée chez lui , il a estimé que la gouvernance doit avoir une tyrannie, et la tyrannie a deux types chez lui: (tyrannie absolue) signifie (le comportement de celui-ci dans la volonté du tout, s'il est d'accord avec la loi ou n'est pas d'accord avec eux, alors c'est lui qui suit le régime et est autorisé à lui seul, et s'il a voulu le faire et s'il ne l'a pas voulu, il n'ya pas de peine sur lui).

Le deuxième type (tyrannie restreinte) signifie (l'indépendance du dirigeant dans la mise en œuvre de loi et de droit décretés après avoir vérifié son approbation autant que possible, et ajoute que ce n'est pas vraiment une simple tyrannie, mais dans la coutume des hommes politiques connue (unicité de l'autorité executive).

La chari'a interdit la tyrannie unique, parce qu'elle a recommandé le principe de la 


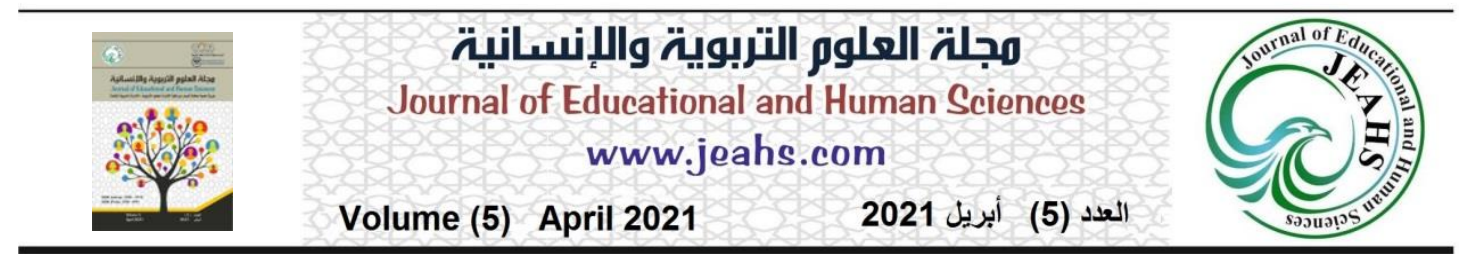

shura, mais la tyrannie restreinte n'est pas interdite dans la chari'a ni dans l'esprit, mais elle est obligatoire, c'est la Shura en particulier, qui est fondée sur ( conseiller les princes) qui est un devoir légitime, mais (comment la conduire ne se limite pas à une certaine manière, le choix de la route spécifique reste sur la permission.)...Mohammed Abdah a souligné l'exemple de la construction de la réforme occidentale et de la nécessité d'adopter ses valeurs d'illumination...

\section{Le phénomène de conflit dans la pensée occidentale entre l'individualité et la collectivité:}

La sagesse d'Allah Le Tout-Puissant dans la création de l'homme y fait apparaitre des qualités apparemment contradictoires, telles que le caractère d'amour avec la haine, de peur avec l'espoir, et la tendance à la réalité avec le sens de l'imagination, et au négatif avec le positif. Et si l'homme fait ces qualités dans le bon champ, Il ne se produit pas entre eux de conflit, mais soit entre eux, ce qui conduit au bonheur de l'homme en ce monde et au-delà. L'une des qualités les plus dangereuses qui paraissent contradictoires est: le sens de l'individualité chez l'homme et le sens de la tendance à se rencontrer, les gens ont divisés à leur égard en trois parties:

Section I: Qui se soucie de l'individu au détriment du collectif.

Section 2: Qui se soucie du côté collectif au détriment du côté individuel.

Section 3: Qui pense qu'il ya la différence entre eux puis a tenté d'y concilier mais dans une fausse manière.

La véritable raison de cette différence est due au manque de compréhension de la vérité de l'âme humaine,et elle ne sera heureuse et droite que si elle prend soin de tous les côtés opposés et donne à chacun sa nourriture appropriée, c'est ce que l'islam offre à l'homme, il nourrit à la fois le côté spirituel et materiel, l'amour et la haine dans son domaine, et la crainte et l'espoir, et ici: comme il nourrit l'individu, il nourrit le collectif et les réconcilie pour le bien l'individu et la société.

\section{Les individualistes:}

Karl Rogers, un psychologue américain, est le pionnier de cette doctrine, quI la dirige vers le domaine de la psychologie et de l'éducation. Il appele pour que les individus aient la possibilité de vivre dans un monde sans pressions de la société, et de choisir les valeurs qui sortent de leur fond. Il veut libérer les individus des contraintes destructrices - comme il les appelle - imposées par la société. La doctrine individuelle est alors une extension des vues de Freud et de l'école de psychanalyse, qui prétendent que l'individu est une victime permanente de la société, et que la société s'impose à l'homme de l'extérieur de son être, elle supprime ses désirs et entrave sa croissance d'origine. 


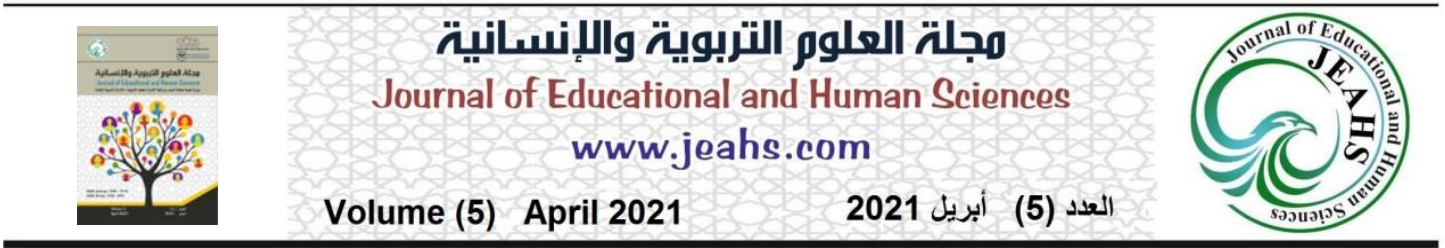

Le capitalisme occidental est basé ainsi sur l'individualité de l'homme, l'étendant dans les limites de son individualité et lui permettant la liberté d'agir beaucoup jusqu'à se faire mal et faire du mal aux autres, sous le slogant de liberté personnelle.

Cette doctrine est également adoptée par les psychologues individualistes, qui considèrent la société d'un point de vue individuel, exagérant son soi en tant que personnalité indépendante, ayant une entité distincte des autres, et exagérant de donner le droit à la société de discipliner l'individu d'obéir. Les existentialists: appellent également à cette doctrine, et les autres qui disent qu'il n'ya de mal pour la société que l'individu se jouit sa liberté individuelle dans ses affaires personnelles, car l'individu est à l'origine, ce qui devrait être réalisé dans sa pleine existence, les individualistes croient que l'éducation doit partir de l'individu et finir avec la société, il faut aider les individus à s'affranchir des restrictions imposées par la société.

Ils croient que les individus se cachent derrière de fausses convictions qui cachent leur vérité fondamentale.Ces fausses convictions sont les valeurs que la société leur a imposé, mais ils croient en effet en d'autres choses, l'éducation doit donc les aider à extraire des valeurs émanant de l'intérieur et briser les restrictions qui leur sont imposées de l'extérieur, pour cette raison les individus traitent les uns avec les autres selon leurs désirs innés, libérés des contraintes tissées par les contraintes de la société. (1)

\section{La conclusion:}

L'individualisme signifie le soucie et la preservation de l'homme en soi, son indépendance et son être, les individualistes voient que l'individu est de tendance individuelle et que la société lui est imposée de l'extérieur, le contrôle hors sa volonté, puis qu'on ne l'aime pas, et que sa fragmentation et sa destruction sont permises (la société), c'est que l'individualisme se fonde à émerger l'existence de l'individu pour le sanctifier, il est interdit à la société de briser sa liberté, et il ne lui est pas permis de l'embarrasser.

L’individualisme a émergé à l'Occident pour plusieurs raisons, notamment:

1-Réaction à la tyrannie de l'Église et son autorité humiliante au peuple.

2-Réaction à la tyrannie des seigneurs féodaux aux gens, leur droits et leurs libertés.

3-Le coup d'État industriel de la Renaissance européenne, qui a entraîné un changement complet de l'image de la société, et que les travailleurs sont venus des campagnes pour leur propre bénéfice ...

( ${ }^{1}$ )Mohammed Qutb, études sur le psychisme humain, dixième édition, Dar El Shorouk, 1993, p. 111-115. 


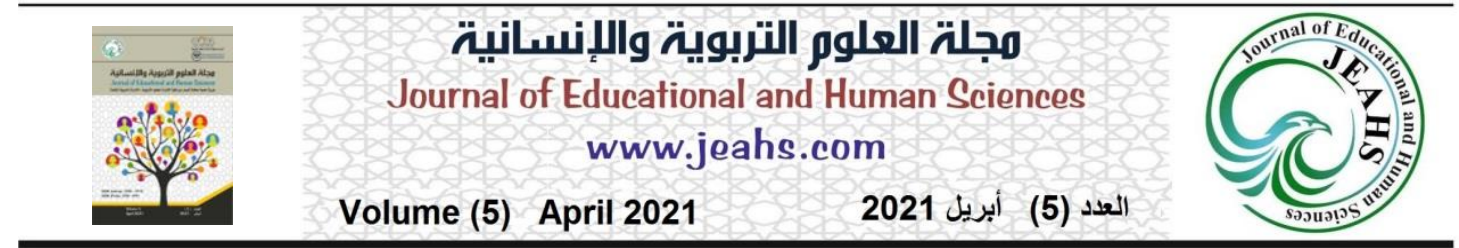

4-L'encouragement du capitalisme pour la doctrine individuelle, qui est basée sur celle-ci, et la défend-elle violemment, en soulignant leur slogan: (Laissez-le travailler. Laissez-le passer), c.-à-d: Laissez l'individu faire ce qu'il veut sans barrière, et passer sans obstacle. C'était un appel à partir des restrictions.

Par conséquent, beaucoup de critiques ont été adressées à cette doctrine, alors la première était de s'intéresser à un côté de l'homme - quelle que soit l'attention - et négligeait un autre aspect, c'est que l'homme n'équilibrait donc pas, et que sa croissance ne serait pas complète, car elle néglige le côté collectif de l'homme, comme un besoin et un désir qui le compose, car chez l'homme ces deux natures, la tendance à l'individualité et la tendance au collectif, deux caractères inhérents qu'on doit être traités ensembles, sinon il y aurait une perturbation à l'intérieur de l'âme et dans la vie réelle. Ils ont étendu le cercle de l'individualité jusqu'à devenir égoïste obscène, et contribuer à briser la société et à disperser ses énergies. Considérant que la société imposée à l'individu est une grande fausse, car la société naît de l'intérieur de l'entité de l'individu, résultant de son désir de rencontrer d'autres personnes. Nous entendons la société: c'est la société naturelle résultant de la convergence d'individus, nous ne voulons pas dire la société déviante, et ils ne le veulent pas, mais ils désignent toute société qui est imposée à l'individu, celle qui entrave sa croissance, et il est vrai qu'il ne s'agit que d'un complément naturel à l'individu et d'une extension naturelle dans laquelle l'individu trouve son être intégré. Alors le départ des individus des traditions correctes de la société sans contrôle, et l'abandon des traditions de la société à cause du déterminisme: c'est l'effondrement d'une société en catastrophe, la frappe de l'intérieur ou de l'extérieur, aboutissant à la fin à priver les individus eux-même de ce qu'ils ont été submergés en désirs permis. Alors la doctrine individuelle nie tout objectif humain loin de l'objectif direct, et nie à l'homme l'élément consistant à regarder au-delà de soi...

C'est donc une fausse clé celui qui ouvre les portes, alors comment faire ce type de clé si elle les ouvre tout le temps? 


\section{مجلة العلوم التربوية والإنسانية}

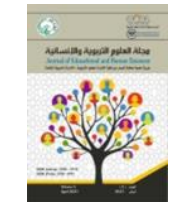

Journal of Educational and Human Sciences

www.jeahs.com

Volume (5) April 2021

العدد (5) أبريل 2021

\section{Références}

1. Ibn Khaldoun, Abd al-Rahman ibn Muhammad ibn Khaldoun, Enquête sur Abdul Wahid Wafi, 4 e s. (Comité de la déclaration arabe du Caire, 1908), 1/364. avait sa proper justification...

2. Jabri, la pensée d'Ibn Khaldoun, la désobéissance et l'État, les caractéristiques de la théorie de Khaldouni dans l'histoire islamique, p.872...

3. Sur l'économie rentière et l'état rentier, voir La nation, l'état et l'intégration dans le monde arabe, de Ghassan Salameh (et al.), C2 Beyrouth: Centre d'études sur l'unité arabe / 1989...

4. Mohammed Qutb, études sur le psychisme humain, dixième édition, Dar El Shorouk, 1993, p. 111-115. 